\title{
Diurnal Variations in Blood Chemical Items in Sprague-Dawley Rats
}

\author{
Sumio MINEMATSU, Masahiko WATANABE, Naoko TSUCHIYA, \\ Masayuki WATANABE, and Sakae AMAGAYA
}

Central Research Laboratories, Tsumura \& Co., 3586 Yoshiwara, Ami-machi, Inashiki-gun, Ibaraki 300-11, Japan

\begin{abstract}
Diurnal variations in 23 blood chemical items were investigated in SpragueDawley rats of both sexes at 6 weeks of age. Blood samples were obtained every two hours. One to three periodic components detected by the maximum entropy method from the observed time-series and the optimum fitting parameters were calculated by the least squares method. The fitting curves were compared with the observed data for the evaluation of systematic and fluctuating parts of the time domain as circadian variations. The periodic components were detected from all the blood chemical items examined. This suggests that all items have systematic parts as basic rhythmic changes. The optimum fitting curves corresponded to the observed time-series in the following items: glucose, total protein, A/G ratio, alkaline phosphatase, $\mathrm{Na}, \mathrm{K}, \mathrm{Cl}$ and $\mathrm{BUN}$ of both sexes and total cholesterol, triglyceride, cholinesterase, CPK, inorganic phosphorus, direct and total bilirubin, creatinine and uric acid in males. Others showed insufficient correspondence to many fluctuating parts. This randomness was more frequently seen in females than in males.
\end{abstract}

Key words: blood chemistry, diurnal variation, rat, time-series analysis

\section{Introduction}

It is well known that many vital phenomena follow a circadian rhythm $(24 \pm 4$ hour cycle $)[3,14]$. Therefore the clock time at operations on animals must be taken into consideration to make the experiment meaningful. The blood chemical examinations are frequently performed as the indicators of the pharmacologic or toxic action of drugs or chemicals. Some items in blood chemistry have been reported with relation to the circadian changes in their blood levels [2, 4, 8, 13]. However, many items of blood chemistry are often measured simultaneously, especially in general toxicity studies. We, therefore, performed the examination to investigate the diurnal variations in 23 blood chemical items as basic in obtaining an understanding of the physiological meaning of observed values. 


\section{Materials and Methods}

Male and female Crj: $\mathrm{CD}$ (SD) rats were purchased from Charles River Japan, Inc. at 5 weeks of age on arrival. After one week's acclimation, they were used at 6 weeks old. The rats were housed in an air-conditioned animal room kept at a temperature of $23 \pm 2^{\circ} \mathrm{C}$ and a relative humidity of $55 \pm 10 \%$. A 12 hour dark/ light cycle was used throughout the experiments (light period, 6:00-18:00). Filtrated tap water and a commercial diet (CRF-1, Oriental Yeast) were available ad libitum.

Blood samples were taken from 8:00 a.m. to 8:00 a.m. the next morning at a $2 \mathrm{hr}$ interval. The blood collection was performed via the vena cava under ether anesthesia. After collection, the blood was kept in an ice cold chamber for 30 to $45 \mathrm{~min}$. Serum samples were then taken by centrifugation $(3,000 \mathrm{rpm}, 10 \mathrm{~min})$ and were frozen below $-20^{\circ} \mathrm{C}$ until analysis. Feeding and drinking were not restricted. At each sampling point, seven rats were used.

The following 23 items in the serum were analysed with an auto-analyser (TBA-60R, Toshiba Medical): glucose (GLU, glucokinase - G-6-PDH method), total cholesterol (CHOL, COD - DAOS method), triglyceride (TRIG, GPO - DAOS method), total protein (PRO, Biuret method), albumin (ALB, BCG method), albumin-globulin ratio $(A / G$, calculation $[A / G=A L B /$ (PRO-ALB)]), glutamic oxaloacetic transaminase (GOT, UV (JSCC) method), glutamic pyruvic transaminase (GPT, UV(JSCC)method), alkaline phosphatase (ALP, PNPP method), lactic dehydrogenase (LDH, Wr ôbiewski-La Due method), cholinesterase (ChE, DTNB method, acetylthiocholine was used as the substrate), creatine phosphokinase (CPK, UV (GSCC) method), sodium( $\mathrm{Na}$, ion selective electrode method), potassium $(\mathrm{K}$, ion selective electrode method), chlorine $(\mathrm{Cl}$, ion selective electrode method), calcium ( $\mathrm{Ca}$, OCPC method), inorganic phosphorus (IP, molybdenum blue method), iron (Fe, BPT method), direct bilirubin (DBIL, alkaliazobilirubin method), total bilirubin (T-BIL, alkliazobilirubin method), urea nitrogen (BUN, urease GIDH method), creatinin (CREA, Jaffé method) and uric acid (UA, uricase POD method).

There are many phenomena which change with time and the data obtained from them with constant time intervals are a so-called time-series. The analysis of a time-series reveals to us the information included in the phenomenon [7]. The mean values at each sampling point were therefore calculated for each item and they were regarded as a time-series for analyses. The flowchart of time-series analysis is shown in Fig. 1. Time-series data $x(t)$ at time $t$ could be shown as

$\mathrm{x}(\mathrm{t})=$ systematic part + fluctuating part

where the systematic part is constructed of periodic components and the fluctuating part means random changing [7]. Periodic components were therefore detected from the original time-series data by using the maximum entropy method (MEM) to determine the systematic part. Thereafter the optimum fitting parameters (crest, amplitude and mesor) were calculated by least square fitting (LSF) with one to three observed periodic components. The fluctuating part was then evaluated by visual inspection of the fitness of the optimum fitting curve with the observed time-series. Though the time lag in MEM analysis could not be determined uniformly, it is known by experience that a

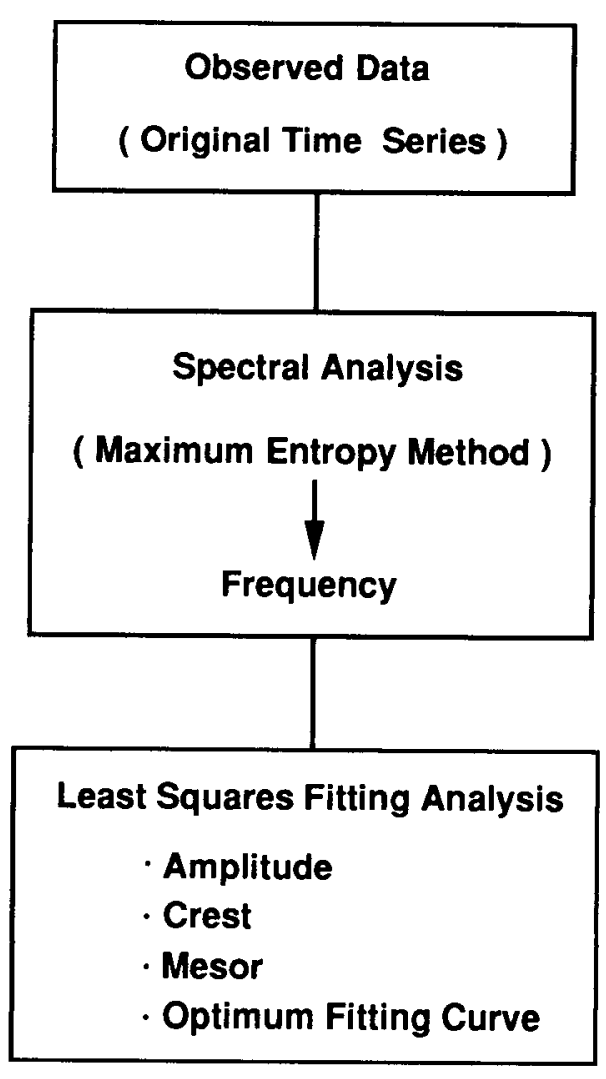

Fig. 1. Flow chart of the time-series data analysis. 
stable spectrum could be obtained if the selected time lag was between $1 / 3$ and $1 / 2$ of the sampling points [6]. The lag was therefore set at 6 , about half of the sampling points, in this study. These analyses were performed by means of a personal computer based on time-series data analysis (MemCalc 1000, Suwa Trast, Tokyo) [9].

\section{Results}

The obtained maximum and minimum values and their ratios for 23 items are shown in Table 1.

\section{Glucose and Lipids (Fig. 2)}

The serum level of GLU was higher during the dark period than the light period, whereas TRIG was lower during the dark period than the light period in both sexes. On the other hand, CHOL did not show an obvious diurnal variation in the observed data. From the LSF analyses, the GLU mesors were almost the same in both sexes and the optimum fitting curves corresponded to the observed time-series. The CHOL mesor in males was slightly higher than that in females. The fitness of the optimum fitting curve and observed time-series in males was better than that in females. TRIG also showed a higher mesor level and better fitting in males than in females. TRIG in females did not show sufficient fitting.

The largest differences between the observed diurnal maximum and minimum values were seen in the TRIG of both sexes in which ratios were over $200 \%$, whereas

\section{Male}

\section{GLU}
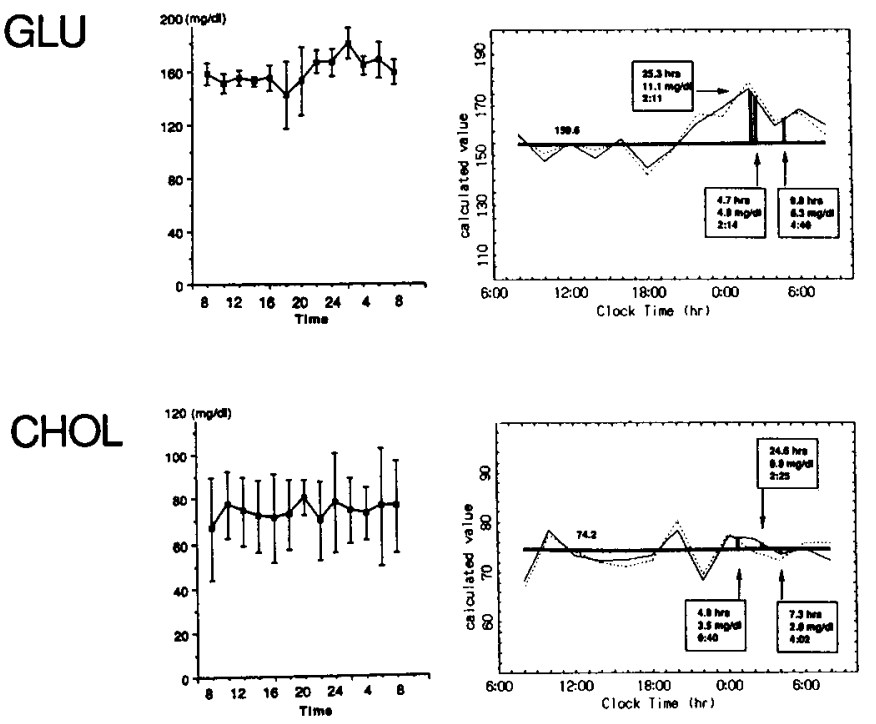

TRIG
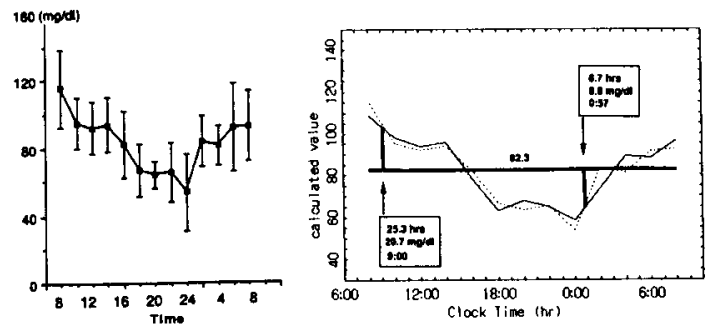

\section{Female}
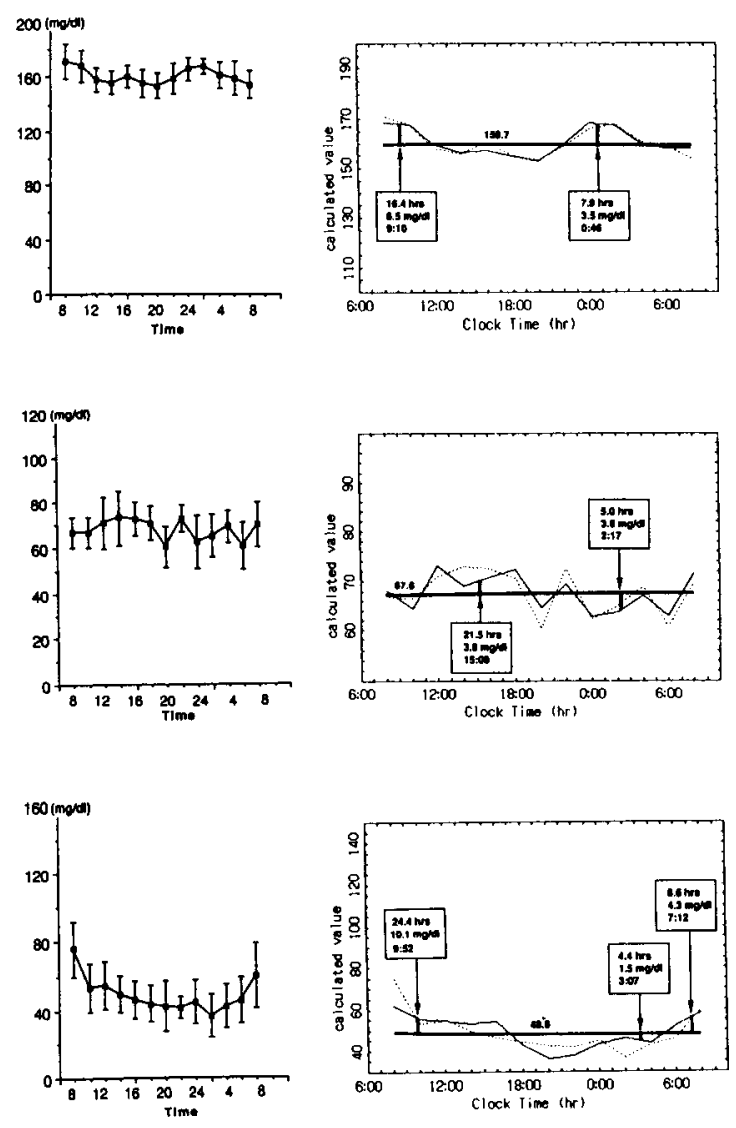

Fig. 2. Diurnal changes in glucose and lipids in males (left columns) and females (right columns). The observed data represents the mean \pm S. D. (left panel in each colum). The optimum fitting curves (solid lines) and observed time-series (dotted lines) are shown in the right panels. Figures in the rectangle in the right panels represent frequency, amplitude and crest, respectively. The horizontal segment of solid line and the numeral above it show the mesor. 
in GLU and CHOL there were not such large changes in absolute values (Table 1).

\section{Proteins (Fig. 3)}

In males and females both PRO and ALB levels were higher during the dark period than the light period. On the other hand, $A / G$ in males was lower during the light period than the dark period, but in females $A / G$ was the highest at 22:00 and thereafter the values began to decrease gradually. The optimum fitting curves of $\mathrm{PRO}$ and $\mathrm{A} / \mathrm{G}$ corresponded to the observed data for each in both males and females, but the ALB curve showed unsatisfactory fitting. The mesors of PRO, ALB and $A / G$ in males were slightly higher than those in females.

The observed values for PRO, ALB and A/G did not show any marked differences during the day (Table 1).

\section{Enzymes (Fig. 4)}

GOT showed a tendency to a high serum level during the dark period and a low level during the light period. On the other hand, the serum level of GPT was lower during the dark period than the light period. The ALP level fell gradually during the light period and then began to increase at the onset of the dark period (18:00). LDH had enormous amplitude and showed signs of changes independent of the light/dark cycle. ChE was roughly at a high level during the dark period and low level during the light period. CPK in males reached its highest value at the onset of the dark period (18:00). The changes in CPK in females were not so large, but a tendency to a high level during the light period and a low level during the dark period was observed. From LSF analyses, the optimum fitting curves

\section{Male}

PRO
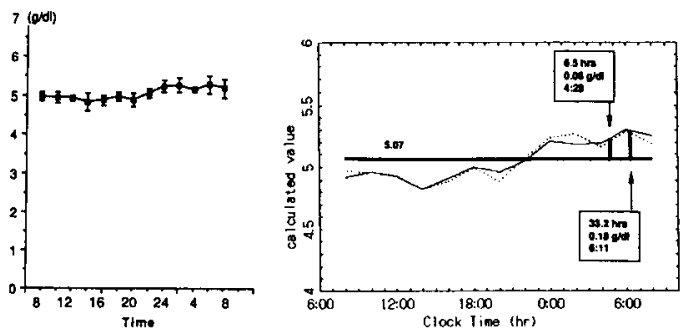

ALB
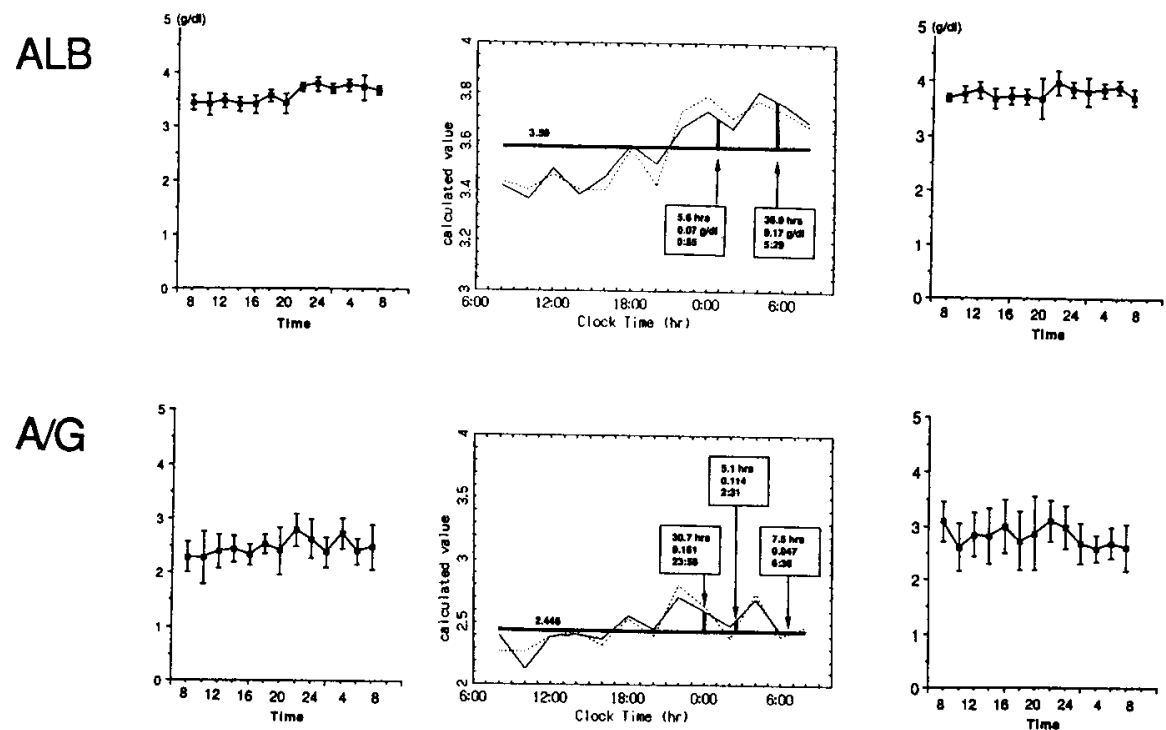

Fig. 3. Diurnal changes in proteins. See the legend to Fig. 2.
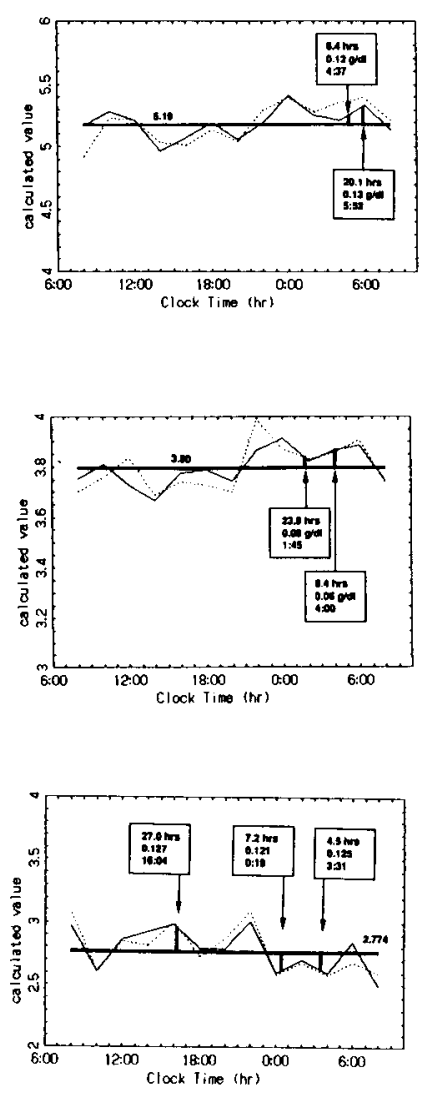


\section{Male}
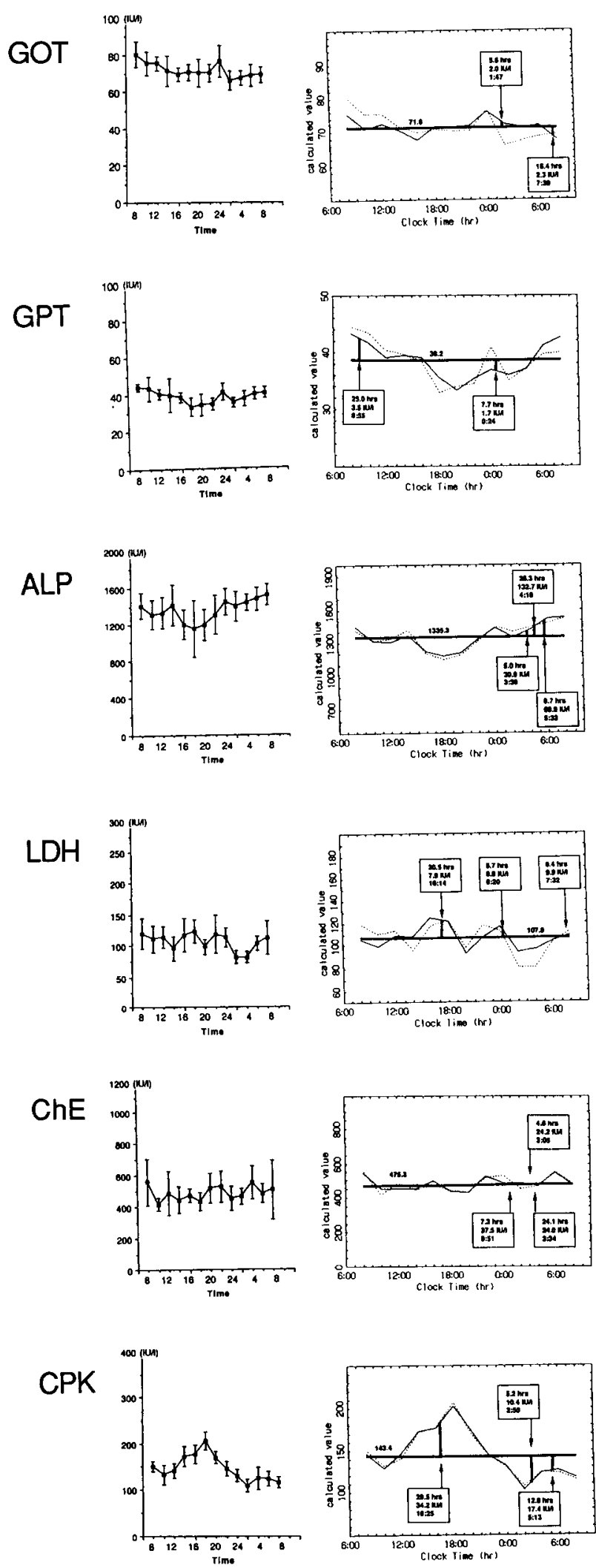

\section{Female}
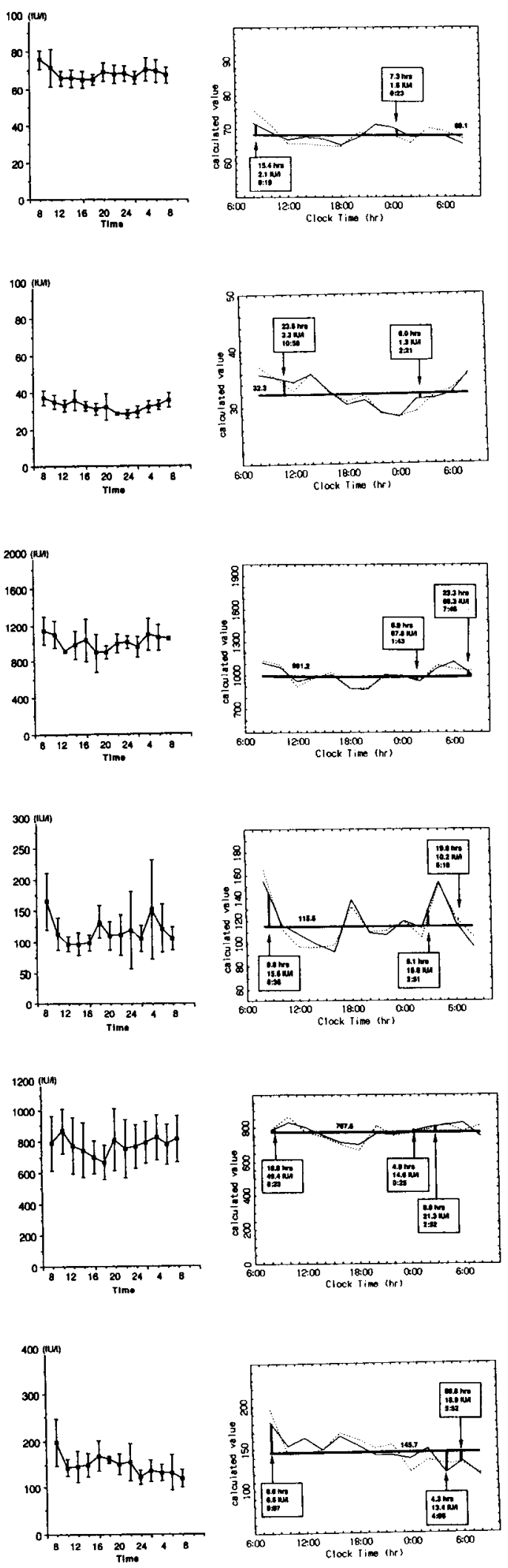

Fig. 4. Diurnal changes in enzymes. See the legend to Fig. 2. 
of GOT, GPT and LDH in males and CPK in females showed insufficient coincidence with each observed time-series. The GOT, GPT and ALP mesors in males were slightly higher than those in females and those of LDH and CPK in females were higher than those in males. In ChE, the mesor in females was markedly higher than that in males.

Observed diurnal maximum and minimum values for LDH and CPK of both sexes differed over $150 \%$ (Table 1).

\section{Inorganics (Fig. 5)}

$\mathrm{Na}$ and $\mathrm{Cl}$ were at a lower level during the light period than the dark period in both males and females. On the other hand, K, Ca and IP were higher during the light period than the dark period. Fe fluctuated greatly and reached its lowest level at the onset of the dark period. The Fe level then began to increase gradually and reached its peak immediately before the end of the dark period, followed by a decrease. From the LSF analyses, the optimum fitting curves for $\mathrm{Na}, \mathrm{K}$ and $\mathrm{Cl}$ showed sufficient coincidence with the observed data in both sexes. However, $\mathrm{Ca}$ in males and females and IP in females did not fit sufficiently, and $\mathrm{Fe}$ in males and females showed almost no fitting. The mesors were almost the same values in males and females except for Fe which was higher in females than in males.

Only the Fe of both sexes showed marked differences between maximum and minimum values (Table 1).

\section{Liver and kidney related items (Fig. 6)}

Both D-BIL and T-BIL in males reached low levels during the light period. From the onset of the dark period, they began to increase, and at the end of the dark period they reached the maximum, followed by a reduction. On the other hand, D-BIL and T-BIL in females did not show obvious circadian changes. BUN and CREA in males and females reached the lowest level at the onset of the dark period and then they began to increase. The maximum levels were seen at the end of the dark period. Though the UA level in males was low during the light period and high during the dark period, it was high during the light period and low during the dark period in females. LSF analyses in males showed satisfactory fitting of the theoretical curves with the observed time-series. In females only
BUN showed sufficient fitting. The mesors for every item were almost the same in both sexes.

All items except CREA in this category showed marked diurnal differences in observed values, especially in UA which had a ratio difference of about $300 \%$ between maximum and minimum values (Table 1 ).

\section{Discussion}

The blood chemical items investigated in this study were analysed by MEM which is believed to be the most reliable method for time-series analysis $[6,7,9]$. We therefore succeeded in detecting the periodic components from the time-series which has only 13 sampling points.

Although a circadian rhythm has been observed in the protein fraction [8], ALP [4, 14] and calcium [2] in rats, the results suggest that every item examined in this study has periodic changes as a systematic part since a power spectrum was detected for all 23 items in this study. Furthermore, some items showed marked differences between diuranal maximum and minimum values. This indicates that the sampling time must be taken into consideration in determing the meaning of the observed values.

In many items there were random components which were more frequently seen in females than in males. These phenomena might reflect the sex differences in physiological function. We performed this study to obtain basic data on the diurnal changes in blood chemical items which are measured routinely, so that further examinations will be necessary to fully clarify the physiological meaning. And even for the 23 items examined in this study, more detailed changes with short sampling intervals and the frequencies with long term sampling should be determined in the future. Below are some brief comments on the results.

Glucose and Lipids: The TRIG blood level was opposite to that of GLU, that is the level was high during the light period and low during the dark period. The blood sugar deficiency triggered the release of free fatty acids and glycerol which were decomposition products of TRIG in fatty tissue, and some fatty acids entered the liver to induce the synthesis of TRIG. These pathways and a reduction of the uptake of TRIG by fatty tissue resulted from the increase in the blood TRIG level [10]. From this, the low blood level of GLU as 


\section{Male}

$\mathrm{Na}$
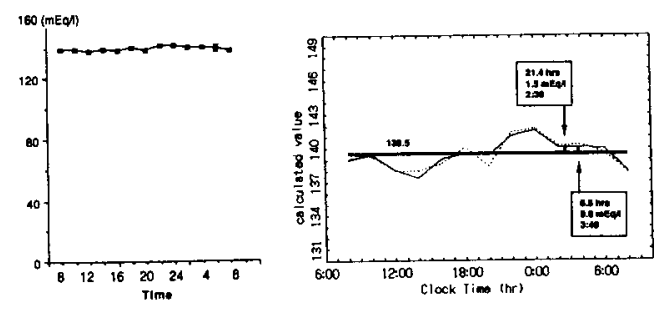

K
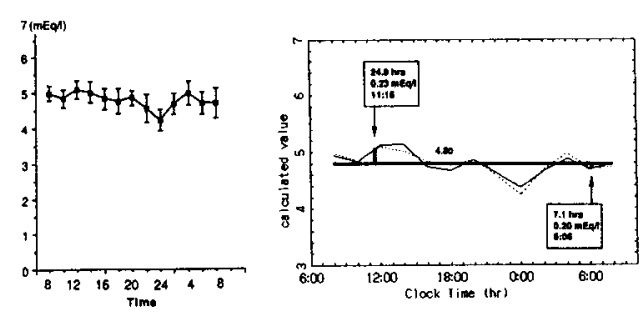

$\mathrm{Cl}$
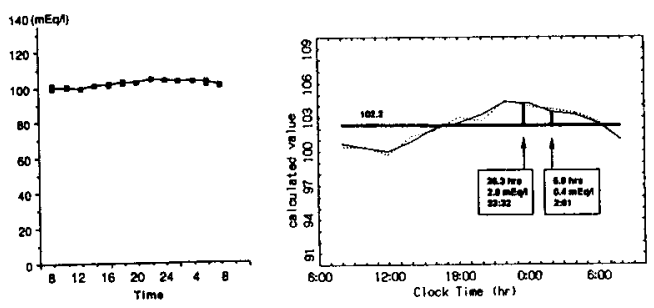

$\mathrm{Ca}$
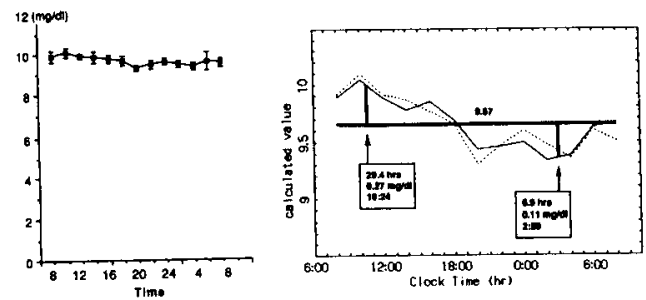

IP
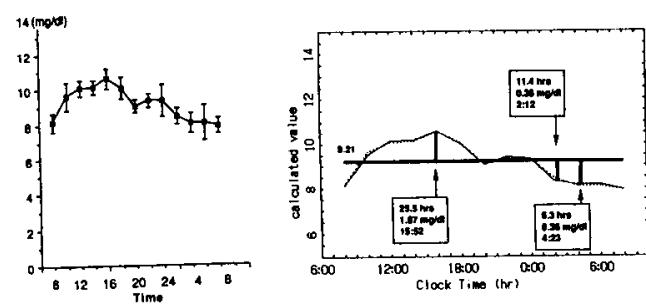

$\mathrm{Fe}$

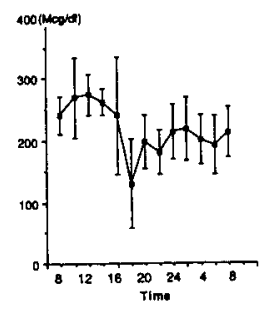

\section{Female}
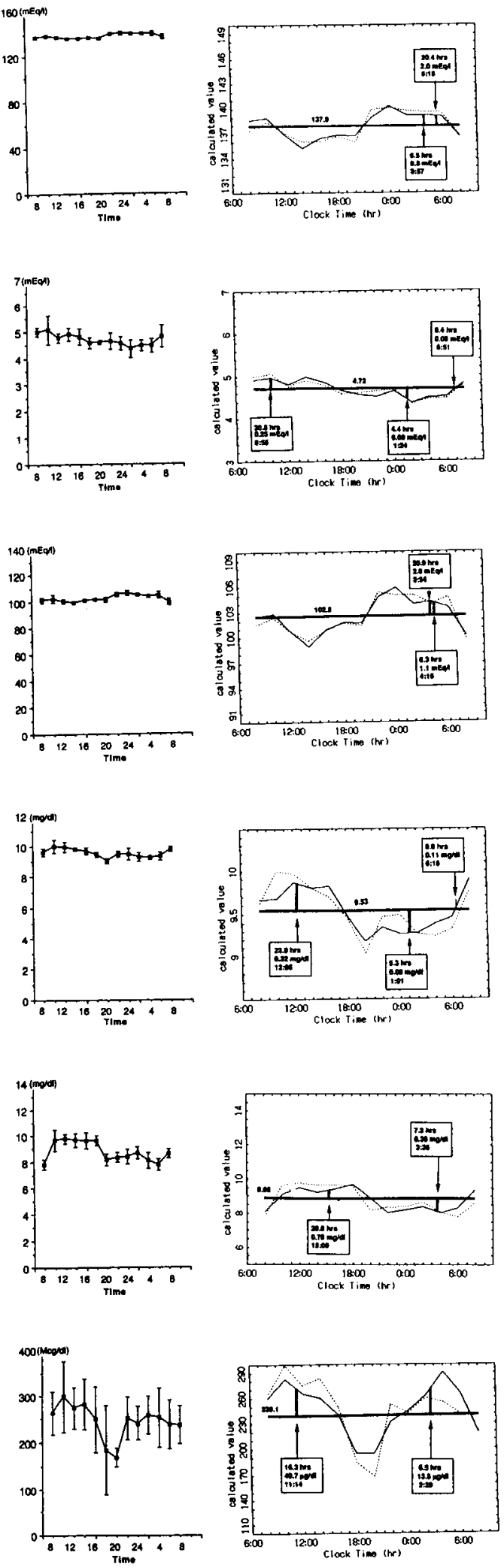

Fig. 5. Diurnal changes in inorganics. See the legend to Fig. 2. 


\section{Male}

D-BIL
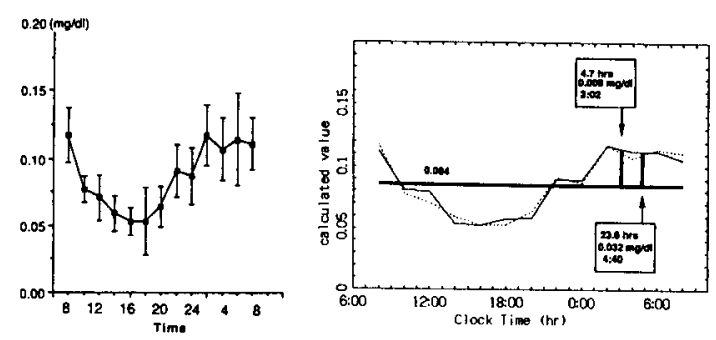

T-BIL
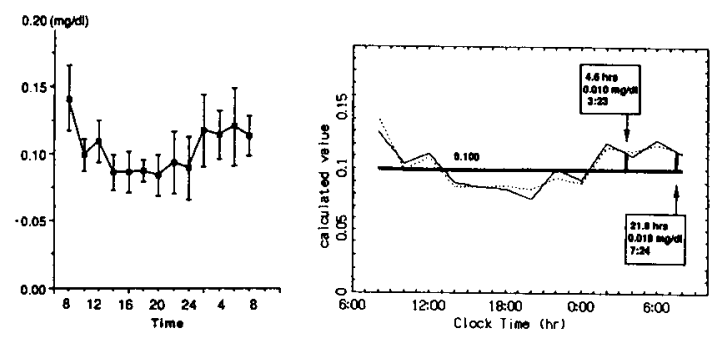

BUN
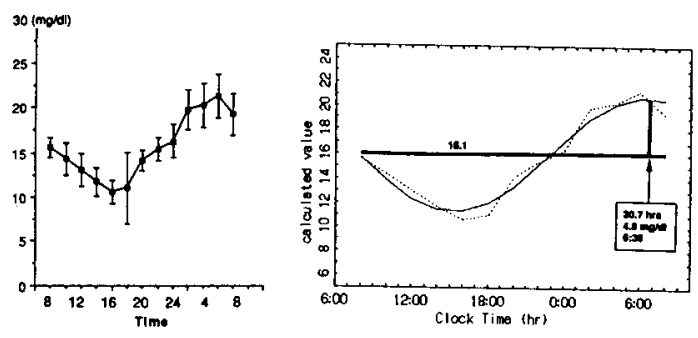

CREA
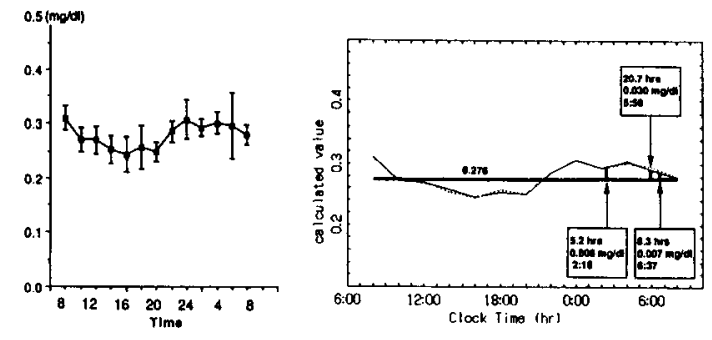

UA
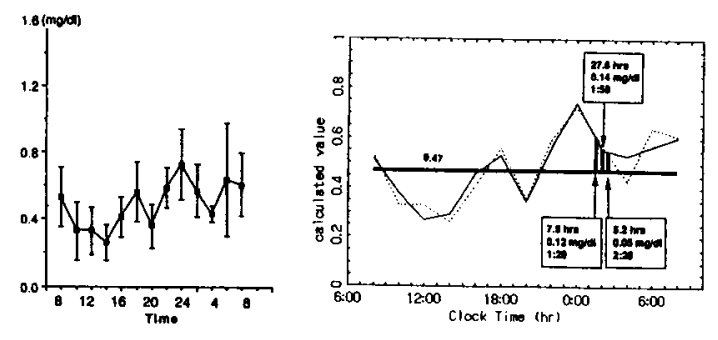

\section{Female}
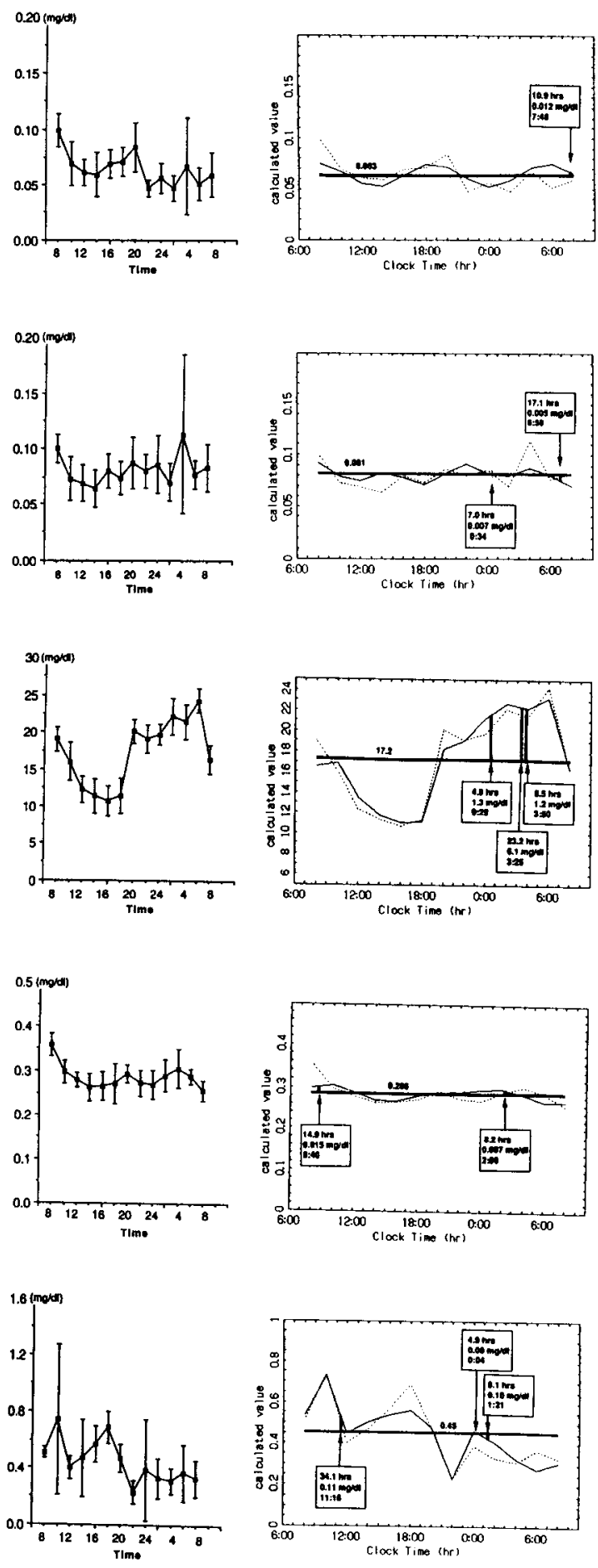

Fig. 6. Diurnal changes in liver and kidney related items. See the legend to Fig. 2. 
Table 1. Diurnal maximum and minimum values for blood chemical items obtained from Sprague-Dawley rats

\begin{tabular}{|c|c|c|c|c|c|c|}
\hline \multirow[b]{2}{*}{ Item (Unit) } & \multicolumn{3}{|c|}{ Male } & \multicolumn{3}{|c|}{ Female } \\
\hline & $\begin{array}{c}\text { Maximum } \\
\text { Mean (A) } \pm S . D .\end{array}$ & $\begin{array}{c}\text { Minimum } \\
\text { Mean (B) } \pm \text { S. D. }\end{array}$ & $\begin{array}{l}\text { Ratio }(\%) \\
(\mathrm{A} / \mathrm{B} \times 100)\end{array}$ & $\begin{array}{c}\text { Maximum } \\
\text { Mean }(A) \pm S . D .\end{array}$ & $\begin{array}{c}\text { Minimum } \\
\text { Mean (B) } \pm \text { S. D. }\end{array}$ & $\begin{array}{l}\text { Ratio }(\%) \\
(\mathrm{A} / \mathrm{B} \times 100)\end{array}$ \\
\hline GLU $\quad(\mathrm{mg} / \mathrm{d} l)$ & $179.7 \pm 11.5$ & $142.0 \pm 25.0$ & 126.5 & $171.1 \pm 12.7$ & $153.3 \pm$ & 111.6 \\
\hline CHOL $(\mathrm{mg} / \mathrm{d} l)$ & $80.3 \pm 16.3$ & $66.7 \pm 6.6$ & 120.4 & $73.1 \pm 12.0$ & $60.4 \pm 8.8$ & 121.0 \\
\hline TRIG $(\mathrm{mg} / \mathrm{d} l)$ & $115.7 \pm 23.1$ & $53.6 \pm 22.3$ & 215.9 & $75.9 \pm 16.0$ & $36.7 \pm 12.6$ & 206.8 \\
\hline$(\mathrm{g} / \mathrm{d} /)$ & $5.29 \pm 0.22$ & $4.83 \pm$ & 109.5 & $5.39 \pm$ & $4.91 \pm$ & 109.8 \\
\hline$(\mathrm{g} / \mathrm{d} l)$ & $3.79 \pm 0.13$ & $3.41 \pm$ & 111.1 & $3.99 \pm$ & $3.69 \pm$ & 108.1 \\
\hline $\mathrm{A} / \mathrm{G}$ & $2.804 \pm \quad 0.307$ & $2.276 \pm$ & 123.2 & $3.096 \pm$ & $2.581 \pm$ & 120.0 \\
\hline GOT $(\mathrm{IU} / l)$ & $80.3 \pm 6.9$ & $66.0 \pm$ & 121.7 & $75.6 \pm$ & $65.0 \pm$ & 116.3 \\
\hline GPT (IU/l) & $44.4 \pm 2.0$ & $32.9 \pm 4.9$ & 135.0 & $37.3 \pm 4.1$ & $28.3 \pm 2.4$ & 131.8 \\
\hline$(\mathrm{IU} / l)$ & $1520.7 \pm 115.7$ & $1152.0 \pm 311.5$ & 132.0 & $1140.6 \pm 158.4$ & $886.1 \pm 211.8$ & 128.7 \\
\hline $\mathrm{LDH} \quad(\mathrm{IU} / l)$ & $123.3 \pm 18.5$ & $80.8 \pm 10.1$ & 152.6 & $165.6 \pm 45.6$ & $96.9 \pm 18.0$ & 170.9 \\
\hline CHE (IU/l) & $557.9 \pm 149.5$ & $414.4 \pm 41.3$ & 134.6 & $865.9 \pm 142.3$ & $665.0 \pm 109.9$ & 130.2 \\
\hline$(\mathrm{IU} / l)$ & $206.4 \pm 19.6$ & $107.8 \pm 13.4$ & 191.5 & $197.9 \pm 49.6$ & \pm 20.2 & 165.3 \\
\hline$(\mathrm{mEq} / t)$ & $141.7 \pm 1.1$ & $137.9 \pm$ & 102.8 & $140.1 \pm$ & $136.0 \pm$ & 103.0 \\
\hline$(\mathrm{mEq} / \mathrm{l})$ & $5.09 \pm$ & $4.24 \pm$ & 120.0 & $5.07 \pm$ & $4.35 \pm$ & 116.6 \\
\hline$(\mathrm{mEq} / \mathrm{l})$ & $104.4 \pm$ & $99.7 \pm$ & 104.7 & $105.3 \pm$ & $99.6 \pm$ & 105.7 \\
\hline$(\mathrm{mg} / \mathrm{d} /)$ & $10.10 \pm$ & $9.30 \pm$ & 108.6 & $10.00 \pm$ & $9.03 \pm$ & 110.7 \\
\hline$(\mathrm{mg} / \mathrm{d} l)$ & $10.60 \pm 0.58$ & $7.90 \pm 0.43$ & 134.2 & $9.76 \pm 0.36$ & $7.79 \pm 0.46$ & 125.3 \\
\hline$(\mathrm{mcg} / \mathrm{d} l)$ & $274.3 \pm 33.5$ & $129.4 \pm 73.2$ & 211.3 & $299.4 \pm 77.3$ & $168.6 \pm 19.5$ & 177.6 \\
\hline D-BIL $(\mathrm{mg} / \mathrm{d} l)$ & $0.117 \pm$ & $0.053 \pm$ & 220.8 & $0.099 \pm$ & $0.047 \pm$ & 210.6 \\
\hline T-BIL $(\mathrm{mg} / \mathrm{d} l)$ & $0.141 \pm$ & $0.084 \pm$ & 167.9 & $0.100 \pm$ & $0.064 \pm$ & 156.3 \\
\hline BUN $\quad(\mathrm{mg} / \mathrm{d} l)$ & $21.3 \pm$ & $10.6 \pm$ & 200.9 & $24.3 \pm$ & $10.7 \pm$ & 227.1 \\
\hline CREA $(\mathrm{mg} / \mathrm{d} l)$ & $0.309 \pm$ & $0.244 \pm$ & 126.6 & $0.357 \pm$ & $0.257 \pm$ & 138.9 \\
\hline $\mathrm{UA} \quad(\mathrm{mg} / \mathrm{d} l)$ & $0.73 \pm$ & $0.26 \pm$ & 280.1 & $0.74 \pm$ & $0.23 \pm$ & 321.7 \\
\hline
\end{tabular}

an energy source during the light period would induce the synthesis of TRIG to supplement energy.

Proteins : The changes in PRO would correspond to the level of ALB since ALB is a major component of serum proteins [5]. However, proteins except ALB would change at different frequencies since $A / G$ did not have a curve parallel to that of ALB.

Enzymes : In males both GOT and GPT had insufficient optimum curves fitting the observed time series, there being many random components. LDH has only low organ-specification [11] and the fitting of theoretical curves with observed data was not satisfactory. The changes in LDH activity therefore suggest that LDH would be released from various organs at various frequencies. It is well known that the blood level of LDH shows varies greatly depending on the sampling and analytical methods $[5,11]$. In this study, the blood was stored in an ice cold chamber immediately after sampling. The influence of platelets therefore seemed to be minimum. The serum was separated 30 to $45 \mathrm{~min}$ after sampling. Accordingly, this might result in great variation, especially in females, but the same procedure was used throughout the series experiments. We therefore considered that the observed data were reliable at least for the detection of periodicity. Though $\mathrm{ChE} \mathrm{did}$ not show marked diurnal variations in males and females, it could be concluded that ChE activity was low during the light period and high during the dark period. Though in females CPK activity did not show marked changes, qualitatively both males and females have the same diurnal variations. Although the cause was unknown, the diurnal variation in CPK activity, at least in males, would be a physiological variation since the optimum fitting curve in males was almost the same as the curve for the observed values.

Inorganics: Because inorganics or electrolytes except for $\mathrm{Fe}$ play important roles in the body in maintaining the normal activity of cells [12], their se- 
rum levels are strictly controlled. In this study, $\mathrm{Na}, \mathrm{K}$, $\mathrm{Cl}$ and $\mathrm{Ca}$ showed obvious circadian changes in both sexes, although the absolute changes were small. IP revealed clear circadian rhythms in both sexes. It has been believed that the suprachiasmatic nucleus ( $\mathrm{SCN}$ ) triggers many circadian rhythms and that cells in the SCN have a circadian rhythm in vitro [1]. The circadian rhythm is therefore a phenomenon which can be observed even in a cell, so that the rhythms of blood levels of electrolytes may correspond to the circadian rhythms of cellular activity. On the other hand, $\mathrm{Fe}$ changed greatly in both sexes. Since the optimum fitting curves were separated from those of observed data, this large change in blood levels in Fe would involve many random components. Further study will be required to clarify this mechanism.

Liver and kidney related items: The increase in the blood levels of BUN at the onset of the dark period would be caused by feeding. CREA showed a little diurnal change and a circadian rhythm in males was clearly indicated. The level of UA activity in males was lower during the light period than the dark period, but the curve for that in females was opposite. It was also noted that the ratio of maximum to minimum UA values was approximately $3: 1$ in both males and females. These sex and diurnal differences suggest that careful handling of UA data is necessary.

\section{References}

1. Aronson, B. D., Bell-Pedersen, D., Block, G. D., Bos, N. P. A., Dunlap, J. C., Eskin, A., Garceau, N. Y., Geusz, M. E., Johnson, K. A., Khalsa, S. B. S., Koster-Van Hoffen, G. C., Koumenis, C., Lee, T. M., LeSauter, J., Lindgren, K. M., Liu, Q., Loros, J. J., Michel, S. H., Mirmiran, M., Moore, R. Y., Ruby, N. F., Silver, R., Turek, F. W., Zatz, M., and Zucker, I. 1993. Circadian rhythms. Brain Res.
Rev. 18:315-333.

2. Lausson, S., Staub, J. F., Milhaud, G., and Perault-Staub, A. M. 1985. Circadian variations in plasma calcium and calcitonin: effect of calcium deficiency and fasting. $J$. Endocr. 107: 389-395.

3. Lemmer, B. 1987. Chronopharmacology: A new perspective in pharmacology. Pol. J. Pharmacol. Pharm. 39: 435-449.

4. Matsumoto, K. 1976. Some problems on the biochemical examinations in rats. Exp. Anim. 25: 229-233 (in Japanese).

5. Meeks, R. G. 1989. The rat. pp.19-25. In: The Clinical Chemistry of Laboratory Animals (Loeb, W. F., and Quimby, F. W. eds. ), Pergamon Press, New York.

6. Minami, S. 1986. Maximum entropy method. pp.166-180. In: Wave Data Processing for Scientific Measurement. CQ Shuppan, Tokyo (in Japanese).

7. Ohtomo, N., Terachi, S., and Tanaka, Y. 1992. Nonlinear time series analysis 1 . A new method of analysis and the theoretical background. Bull. Fac. Engin. Hokkaido Univ. 158: 43-55 (in Japanese).

8. Scheving, L. E., Pauly, J. E., and Tsai, T.-H. 1968. Circadian fluctuation in plasma proteins of the rat. Am. J. Physiol. 215: 1096-1101.

9. Tanaka, Y., Ohtomo, N., and Terachi, S. 1992. Nonlinear time series analysis 2 . The construction of a data analysis system "Memcalc". Bull. Fac. Engin. Hokkaido Univ. 160: 11-23 (in Japanese).

10. Tanimoto, Y. 1988. Glucose. pp.35-45. In: Biochemistry of Blood and Urine in Experimental Animals, Soft Science, Tokyo (in Japanese).

11. Tanimoto, Y. 1988. Serum enzymes. pp.81-102. In: Biochemistry of Blood and Urine in Experimental Animals. Soft Science, Tokyo (in Japanese).

12. Tanimoto, Y. 1988. Serum electrolytes. pp.103-118. In: Biochemistry of Blood and Urine in Experimental Animals, Soft Science, Tokyo (in Japanese).

13. Thompson, C. S., Mikhailidis, D. P., Gill, D. S., Jeremy, J. Y., Bell, J. L., and Dandona, P. 1989. Effect of starvation and sampling time on plasma alkaline phosphatase activity and calcium homeostasis in the rat. Lab. Anim. 23: 53-58.

14. Wollnik, F. 1989. Physiology and regulation of biological rhythms in laboratory animals: an overview. Lab. Anim. 23: $107-125$. 